\title{
Development and Characterization of a High Precision Vibratory MEMS Gyroscope System with Low-Noise Integrated Readout and Control Electronics
}

\author{
Köhler, D. ${ }^{1}$; Hiller. K. ${ }^{3}$; Forke, R. ${ }^{2}$; Konietzka, S. ${ }^{1}$; Pohle, A. ${ }^{1}$; ; Billep, ${ }^{2}{ }^{2}$; Heinz, S. $^{1}$; Lange, A. ${ }^{1}$ \\ ${ }^{1} E D C$ Electronic Design Chemnitz GmbH, Technologie-Campus 1, 09126 Chemnitz, \\ daniel.koehler@ed-chemnitz.de \\ ${ }^{2}$ Fraunhofer ENAS Chemnitz; ${ }^{3}$ Chemnitz University of Technolog , Center for Microtechnologies
}

\begin{abstract}
In this work a MEMS vibratory gyroscope fabricated with a high aspect ratio technology (25:1) and high quality factor $(\mathrm{Q}>90,000)$ in combination with an ASIC for low noise integrated readout $(0.4 \mu \mathrm{V} / \mathrm{rtHz})$, control and compensation electronics will be introduced. The development of the sensor system focuses on a very symmetric design of the mechanical element as well as of the electronics excitation and detection to be able to detect capacitance changes of few aF. The angular rate sensitivity is $0.7 \mathrm{mV} /(\% / \mathrm{s})$ at the first analog stage. Measurement results of the start-up behavior drive amplitude and frequency stability as well as angular rates will be shown.
\end{abstract}

Key words: MEMS, Gyroscope, ASIC, readout electronics, high precision

\section{Introduction}

In today's modern equipment and devices various micro-electro-mechanical systems (MEMS) in combination with specific electronics for readout and control are used. Lower cost as well as improved technologies, reliability and performance enable a strong growth in the number of applications in this field. In addition there are advantages with respect to traditional sensor systems in terms of lower energy consumption and space requirements which allow measurements very close to the point of interest. MEMS gyroscopes for a precise measurement of angular rates in a range of $\% \mathrm{~h}$ up to $\%$ have high requirements regarding the capabilities of the electronic system components for readout, control and calibration.

\section{Double-decoupled high Q-factor single axis MEMS gyroscope}

The silicon vibratory gyroscope has been designed fully symmetric to reduce the impact of parasitics and disturbances. The mechanical structure is doubly decoupled which means that drive and sense modes are decoupled from each other. For the design of the MEMS sensor a robust design method has been used. The influences of technological tolerances, e.g. wedge angle, mask undercut or equipment specific asymmetric etch profiles, have been included in the design process. With the tolerance analysis, the key elements of the sensor were designed to be insensitive regarding technological tolerances. In particular a stable frequency spacing of the drive and sense mode resonant frequencies could be realized regardless of the absolute frequencies, e.g. after etching with different process parameters.
The MEMS sensors have been fabricated using the BDRIE (Bonding and Deep Reactive Ion Etching) technology [1]. Only one precise anisotropic etch step with perpendicular sidewalls and high aspect ratio $(25: 1)$ defines the active structure of the resonator. The glass-Silicon-glass compounds (Figure 1, Figure 2 ) are hermetically sealed in vacuum $\left(<10^{-4} \mathrm{mbar}\right)$ by Anodic bonding.

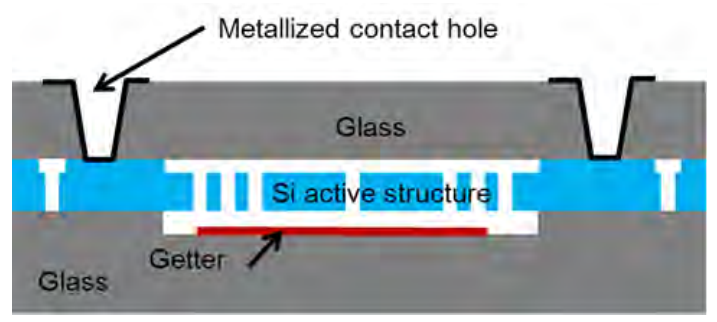

Figure 1: Schematic cross section of the BDRIE sensor

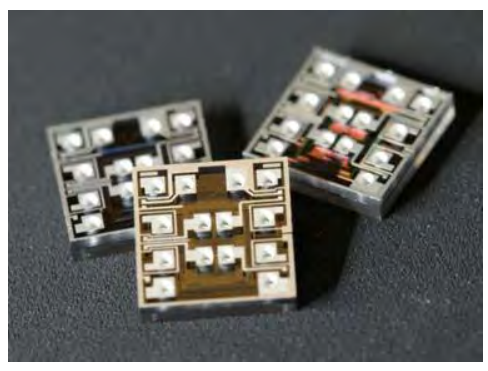

Figure 2: Photograph of MEMS chips

A sputtered thin-film getter layer in the glass cover cavity contributes to a low and long-time stable residual pressure, hereby enabling high $Q$ factors due to reduced gas damping. $Q$ factors of more than 200,000 have been measured for the resonator's drive mode and more than 90,000 for the sense mode. 


\section{Sensor system concept and implementation}

The system will be operated in open loop mode for sense detection and several additional measurement access points are implemented into the system. The MEMS and the ASIC are connected via direct wire bonding to the differential capacitors of sense and drive detection and excitation as well as to the seismic mass and shielding electrodes. The electrodes for sense actuation are already implemented for a possible force feedback solution. The digital signal processing is carried out in an external FPGA board and connected via RS232 interface to PC for analysis in Matlab.

In general the system (Figure 3 ) is based on a MEMS gyroscope and an analog-mixed signal full system ASIC for readout, control and compensation. For drive and sense detection two identical readout chains are used consisting of only few analog components in order to minimize the noise contribution of each element. A low noise continuous time capacitance to voltage converter [2,3] followed by a variable gain amplifier, a filter unit and a $\Sigma \Delta$ low-pass ADC are the main elements of the readout chain. Furthermore the drive control loop is based on the amplitude-phase detection and control unit, the PLL and the related start-up unit. A compensation unit has been designed in order to minimize the impact of unintended disturbing cross-coupling to the signal paths of drive and sense.

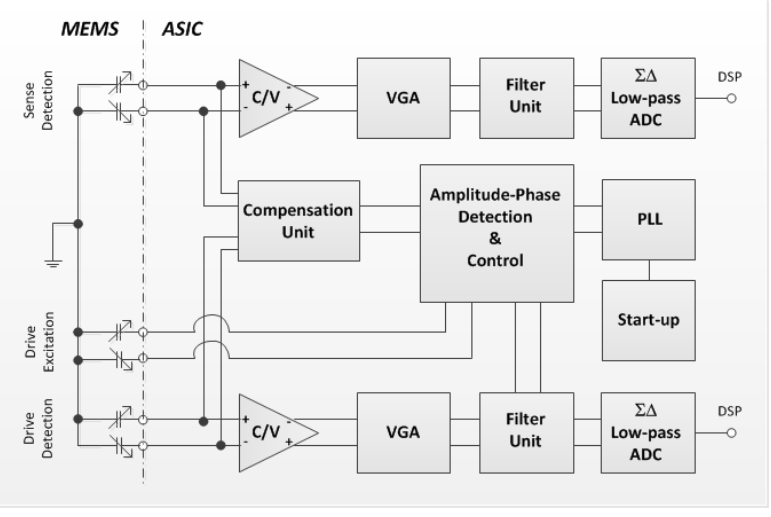

Figure 3: Schematic view- system concept

The MEMS chip is assembled in a CLCC package and the ASIC die is placed directly on top of the MEMS which allow very short and symmetric interconnections (Figure 4). The ASIC is fabricated in a $0.6 \mu \mathrm{m}$ CMOS technology of XFAB Erfurt. The main target specifications for the final system performance are defined in Table 1.

Table 1: Main target specification of the gyroscope system

\begin{tabular}{|c|c|c|}
\hline Parameter & Target Value & Unit \\
\hline Min. Angular Rate & 100 & $\% / \mathrm{h}$ \\
\hline Max. Angular Rate & 500 & $\% / \mathrm{s}$ \\
\hline Bias instability & $<15$ & $\% / \mathrm{h}$ \\
\hline Bandwidth & 120 & $\mathrm{~Hz}$ \\
\hline
\end{tabular}

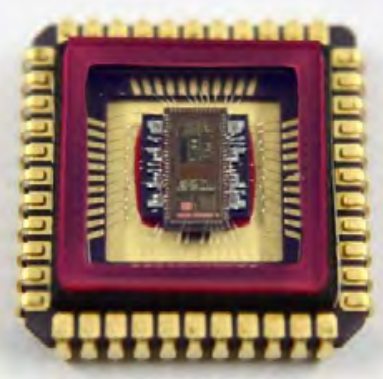

Figure 4: Assembly- ASIC on MEMS package

\section{Measurement results and discussion}

The current operation performance of the gyro system has been evaluated for the cross-coupling compensation (Figure 5, Figure 6) prior the start-up, large angular rates, small angular rates, zero rate test and temperature range. The Allan Variance is used to determine the bias instability and the angle random walk of the gyro system (Figure 7). System outputs during rate table tests are shown in the angular rate plots for small rates (Figure 8) and large rates (Figure 9).

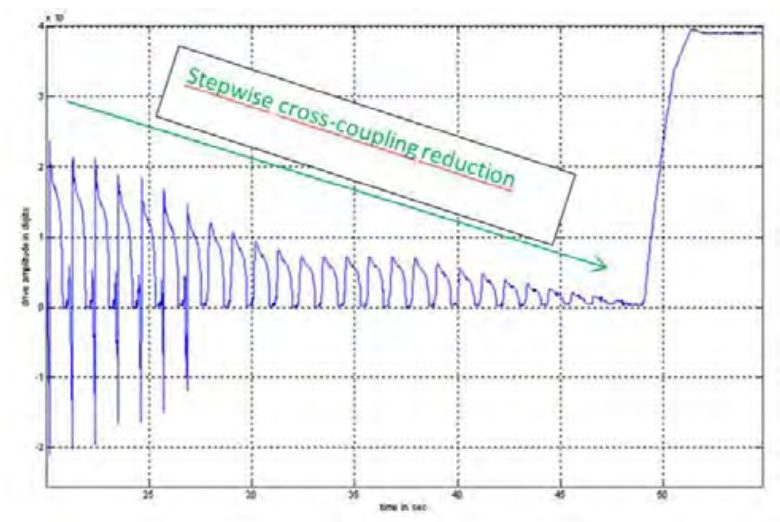

Figure 5: Stepwise Cross-coupling compensation prior startup- Drive amplitude

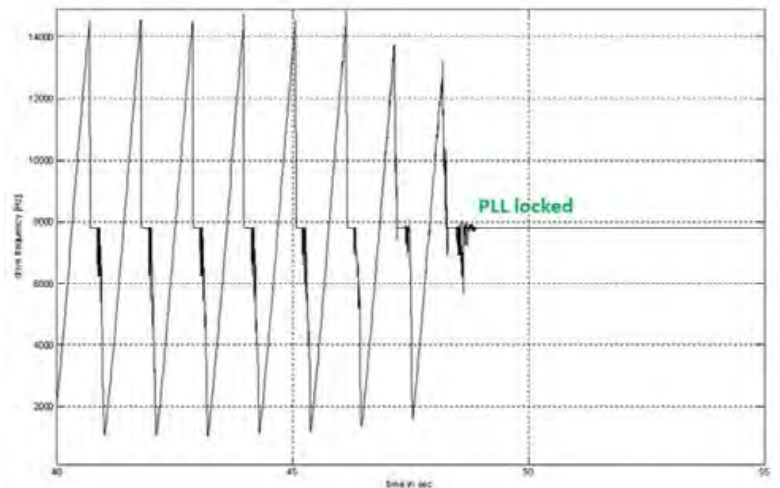

Figure 6: Stepwise cross-coupling compensation prior startup- drive frequency

After a successful compensation procedure the drive resonator starts to oscillate and the PLL locks in. The system can now be switched on/ off reliable and repeatable at the operation point. 


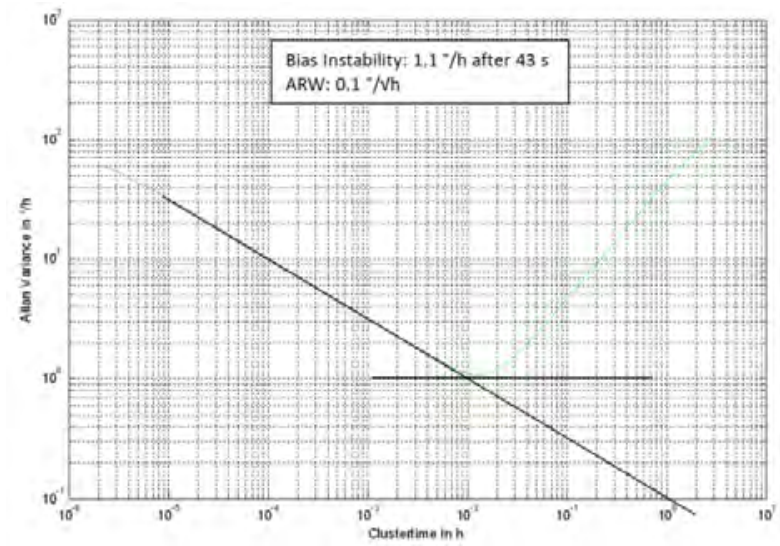

Figure 7: Allan Variance plot

The Allan Variance has been calculated from a 15 hours zero rate measurement with a sampling time of $4 \mathrm{~ms}$.

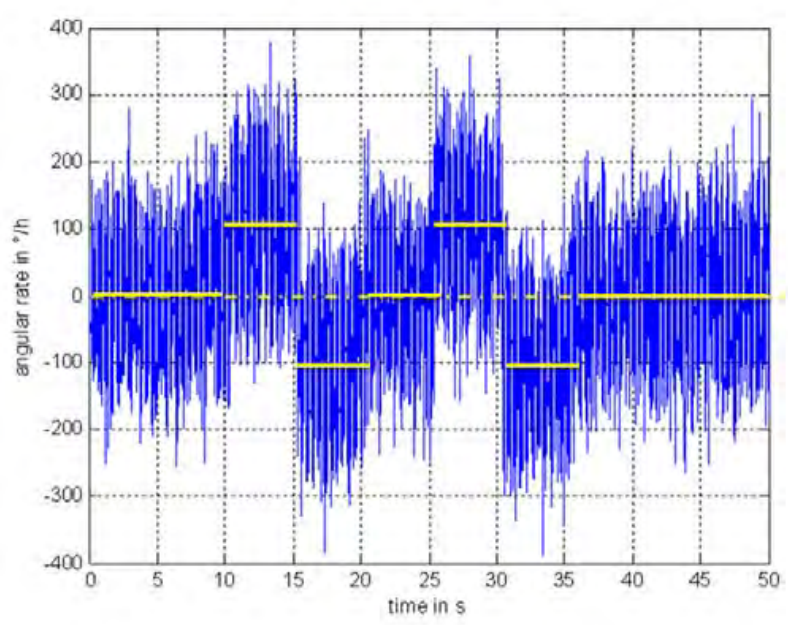

Figure 8: Angular rate plot- small rate

The angular rate output has been evaluated using different methods of compensations and the related relative measurement error. The compensation is done by the application of a phase shift to the sampling of the sense signal in the digital signal processing. The phase shift can be adjusted with respect to the zero rate offset (ZRO) of the angular rate or to a minimum impact of an angular rate change to the quadrature channel.

A relative measurement error of less than $0.8 \%$ fullscale could be achieved (Figure 10). Both compensation methods can be used with a small advantage for the ZRO compensation method.

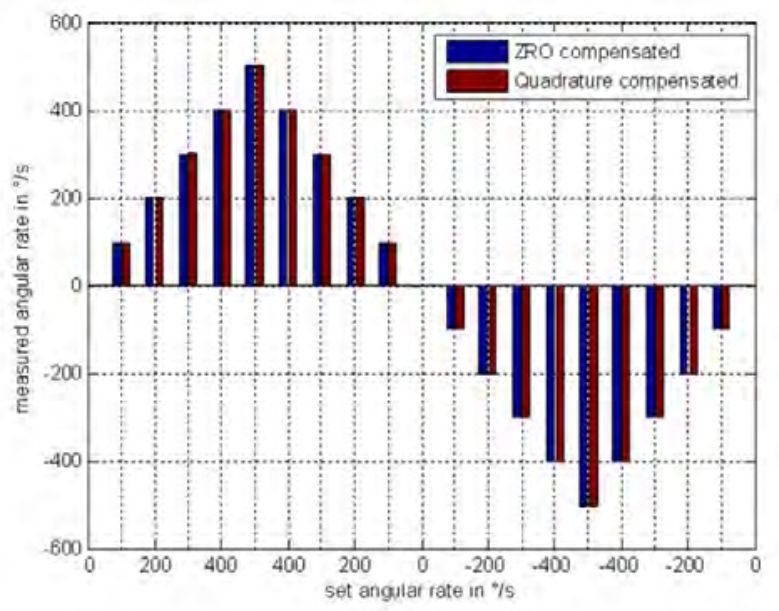

Figure 9: Angular rate plot- large rates

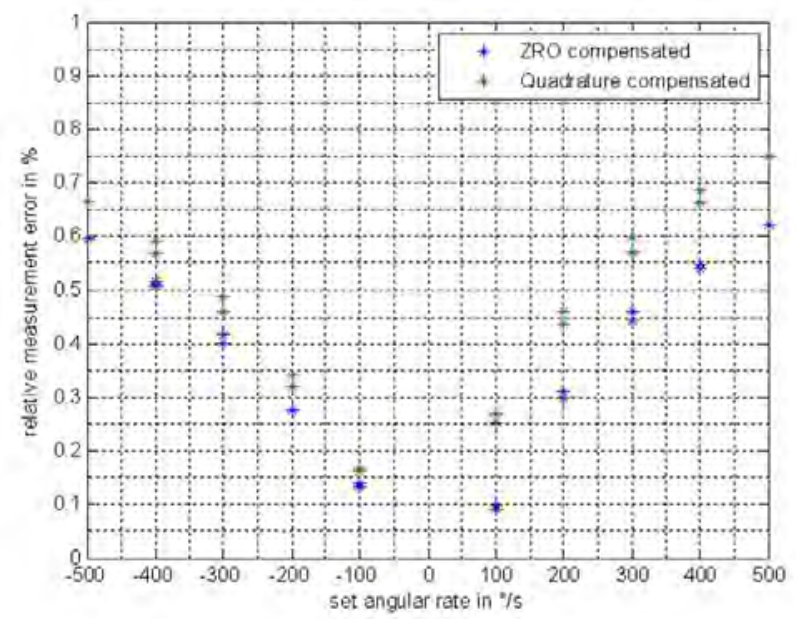

Figure 10: Relative measurement error

\section{Summary}

Although the early stage of the implantation remarkable results with this open loop system could be already achieved with a bias instability of almost $1 \% \mathrm{~h}$, a wide measurement range $0.03-500 \%$ and a relative measurement error less than $0.8 \%$ full-scale.

\section{Literature}

[1] K. Hiller et al.: 'Bonding and Deep RIE - a powerful combination for high aspect ratio sensors and actuators', Proc. of SPIE Vol. 5715, San Jose (CA), 2005

[2] Mikko Saukoski, Lasse Aaltonen, Kari Halonen: 'Integrated Readout and Control Electronics for a Microelectromechanical Angular Velocity Sensor;'Solid-State Circuits Conference, 2006. ESSCIRC 2006.

[3] Konietzka, S.: Entwicklung einer integrierten Schaltung zur Auswertung und Ansteuerung eines mikromechanischen Drehratensensors, Diplomarbeit TU Chemnitz, Fakultät für Elektrotechnik und Informationstechnik Professur für Elektronische Bauelemente der Mikro- und Nanotechnik Mai 2010. 